\title{
Discrimination of malignant and benign breast masses using automatic segmentation and features extracted from dynamic contrast-enhanced and diffusion-weighted MRI
}

\author{
XINHUA JIANG ${ }^{1 *}$, FEI XIE ${ }^{1 *}$, LIZHI LIU ${ }^{1 *}$, YANXIA PENG ${ }^{2}$, HONGMIN CAI $^{3 *}$ and LI LI ${ }^{1 *}$ \\ ${ }^{1}$ Department of Medical Imaging, Collaborative Innovation Center for Cancer Medicine, \\ State Key Laboratory of Oncology in South China, Sun Yat-sen University Cancer Center, Guangzhou, \\ Guangdong 510060; ${ }^{2}$ Department of Radiology, The Third Affiliated Hospital of Sun Yat-sen University, \\ Guangzhou, Guangdong 510630; ${ }^{3}$ Department of Computer Science, School of Computer Science and \\ Engineering, South China University of Technology, Guangzhou, Guangdong 510006, P.R. China
}

Received July 29, 2015; Accepted April 21, 2017

DOI: $10.3892 / 01.2018 .8805$

\begin{abstract}
Magnetic resonance imaging exhibits high sensitivity but low specificity for breast cancer. The present study aimed to investigate whether combining morphology, texture features and kinetic features with diffusion-weighted imaging using quantitative analysis improves the accuracy of discriminating malignant from benign breast masses. In total, 104 and 171 malignant lesions in 205 women were included. Additionally, 13 texture and 11 morphology features were computed from each lesion using a semi-automated segmentation method. To increase prediction accuracy, a newly designed classification model, difference-weighted local hyperplane, was used for statistical analysis of the combined effects of the features for predicting lesion type. The mean apparent diffusion coefficient (ADC) value for each lesion was calculated. Diagnostic performances of morphology and texture features, kinetic features and ADC alone and the combination of them were evaluated using receiver operating characteristics analysis. Malignant
\end{abstract}

Correspondence to: Dr Li Li, Department of Medical Imaging, Collaborative Innovation Center for Cancer Medicine, State Key Laboratory of Oncology in South China, Sun Yat-sen University Cancer Center, 651 Dongfeng East Road, Guangzhou, Guangdong 510060, P.R. China

E-mail: li2@mail.sysu.edu.cn

Dr Hongmin Cai, Department of Computer Science, School of Computer Science and Engineering, South China University of Technology, 281 Wushan Road, Guangzhou, Guangdong 510006, P.R. China

E-mail: hmcai@scut.edu.cn

*Contributed equally

Key words: diffusion-weighted imaging, breast mass, quantitative morphology and texture features, computer-aided diagnosis, difference-weighted local hyperplane lesions had lower mean ADCs than benign lesions. By using 10 -fold cross validation scheme, combined morphological and kinetic features achieved a diagnostic average accuracy of 0.87 . Adding an ADC threshold of $1.37 \times 10^{-3} \mathrm{~mm}^{2} / \mathrm{sec}$ increased the overall averaged accuracy to 0.90 . A multivariate model combining ADC values with 6 morphological and kinetic parameters best discriminated malignant from benign lesions. Incorporating morphology and texture features, kinetic features and ADC into a multivariable diagnostic model improves the discriminatory power of breast lesions.

\section{Introduction}

Dynamic contrast-enhanced magnetic resonance imaging (DCE-MRI) is increasingly used for breast cancer diagnosis as an adjunct to conventional imaging techniques (1). It is used for all stages of management, including detection, diagnosis, pre-operative staging, therapy response monitoring and surveillance (2-6). Breast MRI has demonstrated a high sensitivity, but with the shortcoming of varying specificity, reported from 0.37 to $0.97(3,7)$, which may lead to unnecessary biopsies. Studies have demonstrated that using morphological features in routine clinical practice as additional diagnostic criteria in breast MRI can improve specificity without significantly reducing sensitivity $(8,9)$.

Currently, computer-assisted diagnosis (CAD) systems for breast MRI are increasingly used in clinical practice in order to reduce inter-observer variations in interpretations by facilitating a quantitative and objective evaluation of the images (10), thus shortening the time to diagnosis.

Diffusion weighted MRI (DWI) reflects the movement of water within tissues by measuring the degree of random molecular motion, which is quantified by the apparent diffusion coefficient (ADC) value. Previous studies $(11,12)$ used DWI as an additional tool for diagnosing breast cancer and found that the ADC is significantly lower in malignant tumors than in benign breast lesions or normal tissue. The observed low ADC values associated with malignancy are mainly due to high cell density, which causes increased restriction of the 
extracellular matrix and increased fraction of signal from intracellular water (11). Through combined ADC and dynamic contrast-enhanced kinetics, the diagnostic accuracy of breast MRI has improved (13). Yabuuchi et al (14) reported a high accuracy for enhancing breast masses through a combination of DWI and DCE-MRI features. However, the interpretation of breast DCE-MRI imaging is mainly based on judgments from radiologists; it will be beneficiary to derive discrimination rules provided by CAD. To the best of our knowledge, no published studies have conducted a robust assessment by incorporating morphology and texture features, and kinetic features provided by CAD and ADC into a multivariable diagnostic model for the discrimination of breast masses.

The purpose of this study was to retrospectively investigate whether combining morphology, texture features and kinetic features with ADC using quantitative analysis improves the accuracy for differentiating between benign and malignant breast lesions in MRI.

\section{Materials and methods}

Patients and lesions. In total, 320 patients came to Sun Yat-sen University Cancer Center for clinically indicated bilateral breast MRI between September 2008 and December 2011. The patients' breast MRI examinations were retrospectively reviewed. The MRI studies of 205 women with 275 breast lesions met the following inclusion criteria in the present study: i) MRI was performed using a 1.5-T magnet; ii) both DCE-MRI imaging and DWI imaging sequences were performed; iii) diagnosis was confirmed following pathological analysis subsequent to core-needle biopsy or surgical excision (248 lesions), or lesion stability was confirmed at a minimum follow-up of 2 years (27 lesions); iv) lesions presented as a mass according to the breast imaging reporting and data system MRI lexicon; and v) patients had not received a biopsy or received therapy prior to MRI examination. Of the 320 patients, 23 were excluded for not having a suspicious abnormality on dynamic images, 40 were excluded for non-mass-like enhancement, 16 were excluded because their pathological results were not determined, 20 were excluded for having lesions without a sufficient follow-up period, and 16 were excluded for inadequate fat suppression on DWI images.

MRI image acquisition. Imaging was performed with a GE Signa HDx 1.5-T superconductive magnetic system, using a bilateral, dedicated four-channel phased-array breast coil in the prone position. Standard imaging was performed, including an axial fast spin echo (FSE) T1WI and an axial and sagittal FSE T2WI. Subsequently, DWI images were acquired in the axial planes, prior to gadolinium-based contrast material injection, using: A spin-echo single-shot echo planar imaging sequence; array spatial sensitivity encoding technique (acceleration factor of two); b values of 0 and $800 \mathrm{sec} / \mathrm{mm}^{2}$; fat suppression; 5,000/75 (repetition time msec/echo time msec); $5 \mathrm{~mm}$ section thickness; a 30x30 cm field of view; a 128x128 matrix; $0 \mathrm{~mm}$ section gap; and $130 \mathrm{sec}$ acquisition time.

Subsequently, after one set of unenhanced baseline images, dynamic contrast-enhanced MRI data were acquired using an MRI-specific automatic power injector (Medrad Inc., Pittsburgh, PA, USA) to inject $0.1 \mathrm{mmol} / \mathrm{kg}$ body weight contrast medium gadolinium diethylenetriamine penta-acetic acid (Gd-DTPA), with a hand venipuncture technique at a rate of $3 \mathrm{ml} / \mathrm{sec}$. Saline ( $10 \mathrm{ml}$ at $3 \mathrm{ml} / \mathrm{sec}$ ) was then injected to wash the tube. Dynamic scanning was initiated by simultaneously pushing the high-pressure syringe button and the dynamic scan button. Nine post-contrast sets were acquired. Each sequence was performed in the sagittal plane at $20 \mathrm{sec}$ intervals with fat suppression using: Three-dimensional spoiled gradient recall echo sequence; 5.5/2.6 repetition time msec/echo time msec; $3.4 \mathrm{~mm}$ slice thickness; $15^{\circ}$ flip angle; a $22 \times 22 \mathrm{~cm}$ field of view; a 288x192 matrix; and a $59 \mathrm{sec}$ acquisition time. Subsequently, axial MRI was employed using fat-suppressed enhanced T1WI sequence.

Data analyses. All images were analyzed independently by two radiologists with ten years experience in interpreting breast MRI. They were blinded to the histological results of current patients. The images were assessed independently and any disagreements were resolved by achieving consensus. All lesions were assessed using The Breast Imaging Reporting and Data System (BI-RADS) (15). BI-RADS category 1 (negative) and category 2 (benign) denote an essentially $0 \%$ likelihood of cancer. BI-RADS category 3 (probably benign) assessment is more intuitive and can be recommended in the case of a unique focal finding for which the likelihood of malignancy is $\geq 0 \%$ but $\leq 2 \%$. BI-RADS category 4 (suspicious) and category 5 (highly suggestive of malignancy) describe MRI findings that are suspicious enough to warrant tissue diagnosis. BI-RADS category 6 (known biopsy- proven malignancy) describes MRI findings of biopsy-proven breast cancer for which surgical excision is recommended when clinically appropriate. All images were analyzed on a workstation (Centricity Radiology RA 600 V 7.0; GE Healthcare, Chicago, IL, USA). All quantitative analysis software was written in MATLAB (MathWorks, Natick, MA, USA; http//www.mathworks.com).

Analysis of lesion kinetics. For kinetic analysis, time-signal intensity curves were obtained from manually drawn regions of interest (ROIs), the size of which varied with the size of the enhancing lesion and were chosen to selectively include the area with the strongest enhancement, as identified on the first post contrast subtracted image. The early-phase enhancement rate and the signal enhancement ratio (SER) (16) were quantified by means of an ROI-based determination of lesion signal intensity prior and subsequent to the injection of Gd-DTPA. The early-phase enhancement was calculated according to the enhancement formula $\left(\mathrm{SI}_{1}-\mathrm{SI}_{0}\right) / \mathrm{SI}_{0} \times 100$ (17) and SER was defined as $\left(\mathrm{SI}_{1}-\mathrm{SI}_{0}\right) /\left(\mathrm{SI}_{\text {last }}-\mathrm{SI}_{0}\right)$, where $\mathrm{SI}_{0}, \mathrm{SI}_{1}$, and $\mathrm{SI}_{\text {last }}$ represent the signal intensity in the pre-contrast, the first post-contrast and the last images, respectively.

Analysis of morphological and texture features. Following manual lesion identification, automatic segmentation was undertaken for lesion contours. The segmentation used a novel two-step approach that incorporated fuzzy c-means (FCM) clustering (18) and gradient vector flow snake algorithm (19). The FCM clustering based method was used to obtain initialization for commencement of segmentation while the gradient vector flow snake model was further applied to obtain the exact segmentation (Fig. 1). A total 
A

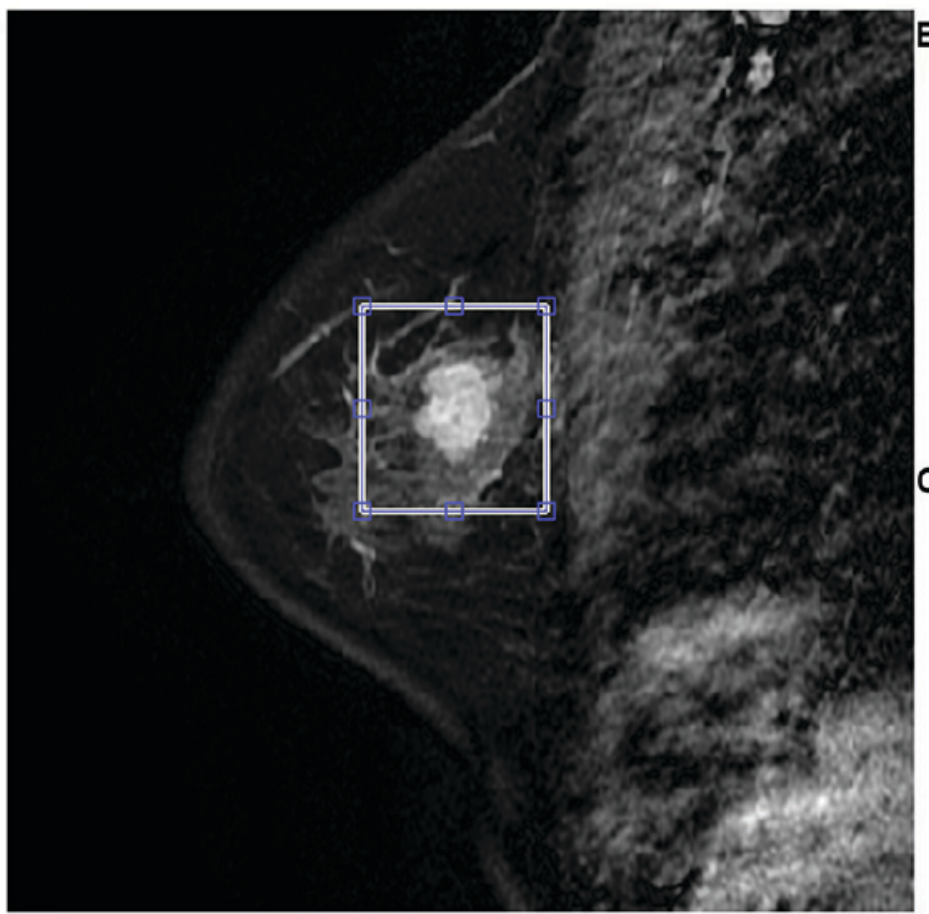

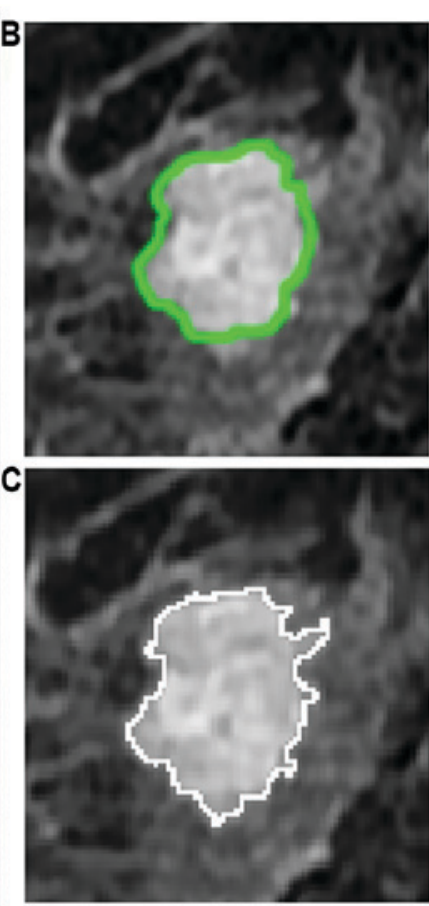

Figure 1. A sample breast lesion detected by magnetic resonance imaging. (A) A post-contrast breast lesion is highlighted in the blue rectangle. (B) Initial segmentation result using the fuzzy c-means-based method. (C) Final segmentation by deformation of gradient vector flow snake with initialization of (B).

of 13 texture features were estimated from the gray level co-occurrence matrix (GLCM) (20). The parameters included: Angular second moment; contrast; correlation; inverse difference moment; sum average; sum variance; sum entropy; entropy; difference average; difference variance; difference entropy; information measure of correlation 1; and information measure of correlation 2 (21). In total, 11 morphological features, including compactness, spiculation, extent, elongation, solidity, circularity, entropy of radial length distribution, fractal, heterogeneity, area and eccentricity, were also computed (22).

Breast masses in the study were all verified histopathologically (Table I), or the diagnosis was confirmed following at $\geq 2$ years of follow-up. Lesion status was used as baseline for statistical evaluation of the performance of the features. The whole patients set was randomly divided into two sets, one for classifier training and the other for testing the performance of the classifier. The ten-fold cross validation scheme was used to evaluate and find the classifier with the best performance.

Analysis of $A D C$. The ROIs were manually drawn on the diffusion-weighted images $\left(b=800 \mathrm{~s} / \mathrm{mm}^{2}\right.$; Figs. 2 and 3$)$ and were placed in regions with high signal intensity on the images. The contrast and morphological characteristics at the early phase of contrast-enhanced T1-weighted imaging and T2-weighted imaging were used to guide ROI placement to prevent areas of T2 shine-through, which was usually found in necrotic or cystic parts. The ROIs were defined as the area slightly smaller than the actual lesions to reduce partial volume effects. In the current study, all ROIs were $>20 \mathrm{~mm}^{2}$ (23). The DWI intensity for each lesion was classified as high or low compared to that of the corresponding background breast tissue. The mean ADC of the voxels in ROIs were then obtained.
Table I. Histopathology of benign and malignant breast lesions.

\begin{tabular}{lrc}
\hline Tumor group & Number & Percentage \\
\hline Malignant lesions & 171 & 62.2 \\
Invasive ductal carcinoma & 136 & 79.5 \\
Intra-ductal carcinoma & 23 & 13.4 \\
Lobular carcinoma & 2 & 1.2 \\
Mucinous carcinoma & 3 & 1.8 \\
Medullary carcinoma & 1 & 0.6 \\
Others & 6 & 3.5 \\
Benign lesions & 104 & 37.8 \\
Fibroadenosis & 64 & 61.6 \\
Intraductal papilloma & 4 & 3.8 \\
Hyperplasia & 4 & 3.8 \\
Phyllodes tumor & 2 & 1.9 \\
Adenomyosis epithelioma & 1 & 1 \\
Inflammation & 2 & 1.9 \\
Follow-up & 27 & 26 \\
\hline
\end{tabular}

Statistical analysis. Morphological features were shown to perform well in terms of discriminating lesion types (8). Meanwhile, using the textural or kinetic measurements alone can also shade light in the characteristics of lesions, shown in Table II. Therefore, the combination of the merits of the characterization parameters, including morphological, textural and kinetic measurements, was expected to achieve a better, or at least comparable performance compared with individual measurements. The combination of the parameters as whole reflected different aspects of lesion properties and was a 
Table II. Group mean, P-values and diagnostic accuracy of selected parameters.

\begin{tabular}{lccccc}
\hline Parameters & Benign $^{\mathrm{a}}$ & Malignant $^{\mathrm{a}}$ & P-value $^{\mathrm{b}}$ & Diagnostic accuracy $^{\mathrm{c}}$ & Threshold value $^{-0.03}$ \\
\hline Heterogeneity & $-0.054 \pm 1.061$ & $0.033 \pm 0.964$ & 0.486 & 0.633 & 0.17 \\
Area & $-0.403 \pm 0.751$ & $0.245 \pm 1.053$ & $<0.001$ & 0.742 & 440.00 \\
Sum variance & $0.373 \pm 1.094$ & $-0.227 \pm 0.866$ & $<0.001$ & 0.684 & 7.88 \\
Sum entropy & $-0.508 \pm 1.057$ & $0.309 \pm 0.825$ & $<0.001$ & 0.709 & 5.06 \\
Difference entropy & $-0.171 \pm 1.112$ & $0.104 \pm 0.914$ & 0.003 & 0.647 & 0.34 \\
SER & $-0.826 \pm 0.756$ & $0.502 \pm 0.771$ & 0 & 0.876 & \\
\hline
\end{tabular}

${ }^{\mathrm{a}}$ Data presented as the mean \pm standard deviation. ${ }^{\mathrm{b}}$ Computed with paired-sample t-test. ${ }^{\mathrm{c}}$ Computed with univariate logistic regression. SER, signal enhancement ratio.

Table III. Diagnostic performance differentiating between malignant and benign lesions.

\begin{tabular}{|c|c|c|c|c|}
\hline Parameter selection & Sensitivity & Specificity & Accuracy & AUC \\
\hline Morphology & 0.57 & 0.78 & 0.70 & 0.68 \\
\hline Kinetics & 0.79 & 0.89 & 0.85 & 0.84 \\
\hline Texture & 0.53 & 0.81 & 0.71 & 0.67 \\
\hline Morphology+texture+kinetics & 0.81 & 0.91 & 0.87 & 0.86 \\
\hline $\mathrm{ADC}$ & 0.78 & 0.90 & 0.84 & 0.83 \\
\hline ADC+kinetics & 0.84 & 0.93 & 0.89 & 0.88 \\
\hline ADC+morphology+kinetics+texture & 0.85 & 0.94 & 0.90 & 0.90 \\
\hline
\end{tabular}

ADC, apparent diffusion coefficient; AUC, area under curve.

potentially comprehensive approach to the characterization of lesion status.

In total, 24 features were initially estimated from the segmented lesion image. Since the features may be self-dependent or irrelevant to the lesion type, an exhaustive feature selection method was used to find an optimal feature subset in which the classification accuracy is highest (24). The differentiation of malignant from benign lesions was treated as a two-class pattern classification problem. A newly reported classification model, the difference-weighted local hyperplane classification model (DWLH), was used to select the feature subset and to evaluate the performance of the combined feature subsets (25). In the classification model, a local hyperplane is constructed from its nearest neighbor for each observed sample. The label of the sample is decided by the minimized distance to its class-dependent hyperplane. The performance of the model is superior to that of the classical k-nearest neighbors algorithm in that the local hyperplane is robust to noises and outliers (26). The present study selected DWLH, not only because of its superior performance over classical models, including the support vector machine, but also as a result of the rationale behind its mathematical formulation (27). Since lesions usually share certain pictorial similarities among patients with the same type lesions, the status of lesion could be deducted from its nearest neighbors, other than particular one as k-nearest neighbors does. This follows the same rationale with the classifier of DWLH.
To test the discrimination power of different characterization of breast masses, the whole dataset where categorized into six subgroups including morphology, kinetics, texture features and their combinations. To further evaluate the discrimination power of ADC, various characteristics of breast masses were combined with/without ADC, resulting another three data subgroups. On each subgroup, the classifier of DWLH was evaluated via ten-fold cross validation scheme. For each experiment, the averaged accuracy and AUC were calculated to serve as criteria of the feature performance. To eliminate the statistical variations during the training phase, the present study conducted 10 classification experiments independently on each dataset. The averaged classification error was recorded and is presented in Table III. Receiver operating characteristic (ROC) analysis was also used to evaluate the diagnostic performance of the model under various features. The area under the ROC curve (AUC) served as the criterion for selecting the best combination.

\section{Results}

In total, 205 women with 275 lesions met the inclusion criteria. The mean age of these patients was $46.2 \pm 10.9$ years (age range, 18-78 years). A total of 48 lesions were assessed as probably benign finding (BI-RADS category 3), 147 were assessed as suspicious (BI-RADS category 4), and 80 were assessed as highly suggestive of malignancy (BI-RADS category 5). Table I exhibits the distribution of histopathological findings 

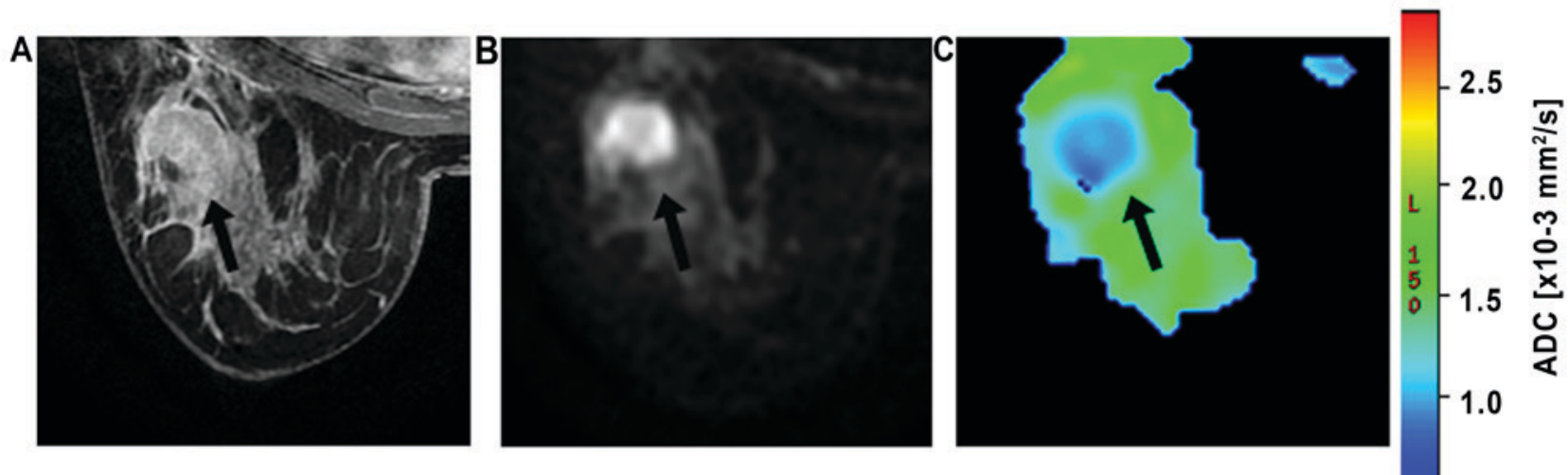

Figure 2. Invasive ductal carcinoma (arrows) in a 30-year-old woman. (A) Dynamic contrast-enhanced MRI image in the early phase. Areas of mass-like enhancement are labeled by arrows and the lesion exhibits high signal intensity. (B) Diffusion-weighted MRI image (b=800 sec/mm ${ }^{2}$ ). $(C)$ ADC map. Lesion exhibits areas with dark blue contours (arrows), which are indicative of a low ADC. ADC measured in this lesion is $0.89 \times 10^{-3} \mathrm{sec} / \mathrm{mm}^{2}$. MRI, magnetic resonance imaging; ADC, apparent diffusion coefficient.
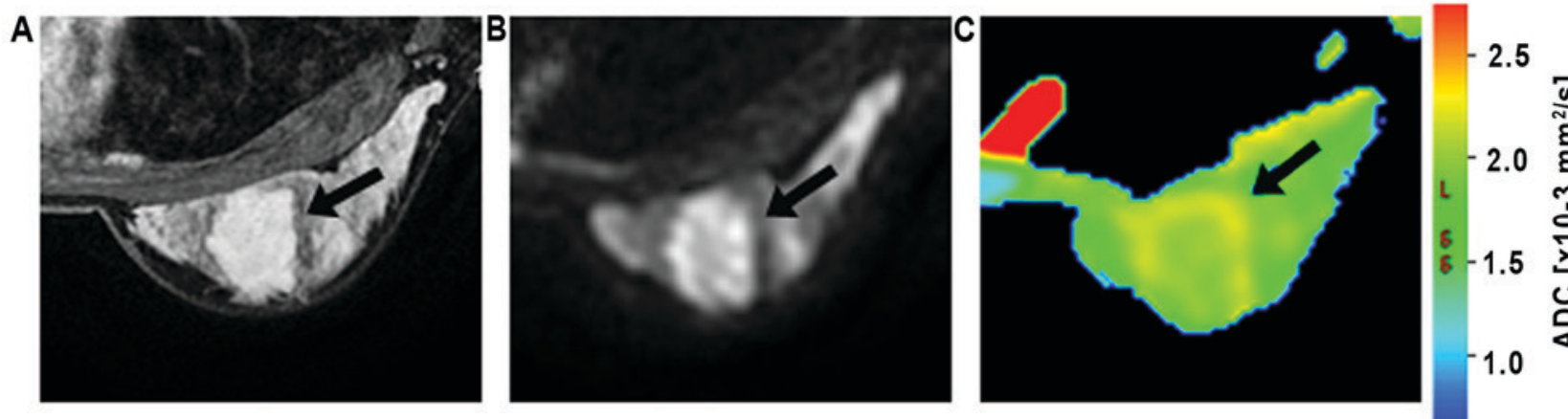

Figure 3. Fibroadenoma detected in an 18-year-old woman. (A) Dynamic contrast-enhanced MR image in the early phase. Areas of mass-like enhancement are labeled by arrows and the lesion exhibits high signal intensity. (B) Diffusion-weighted MRI image ( $\left.\mathrm{b}=800 \mathrm{sec} / \mathrm{mm}^{2}\right)$. (C) ADC map. Lesion exhibits areas with light green contours (arrows), which are indicative of a high ADC. ADC measured in this lesion is $1.91 \times 10^{-3} \mathrm{sec} / \mathrm{mm}^{2}$. MRI, magnetic resonance imaging; ADC, apparent diffusion coefficient.

of all analyzed lesions. The mean lesion size, defined as the longest dimension on DCE-MRI images, was $1.3 \pm 2.1 \mathrm{~cm}$ (range, $0.5-3.0 \mathrm{~cm}$ ) for benign lesions and $2.8 \pm 1.5 \mathrm{~cm}$ (range, $1.5-5.0 \mathrm{~cm}$ ) for malignant lesions.

Diagnostic performance of morphological and kinetic features. Segmentation results of randomly selected sample breast lesion can be seen in Fig. 1. In total, 6 features performed optimally in terms of prediction. The features included two shape (heterogeneity, area) and three texture (sum variance, sum entropy, difference entropy) parameters and one kinetic parameter (SER).

The diagnostic performance of each feature used in the experiment classifier was also evaluated individually. The mean and standard deviation and the diagnostic performance of the 6 selected parameters are summarized in Table II. Among the 6 parameters, the diagnostic accuracy of SER was the highest.

Utilization of 5 anatomical features, including texture and shape parameters, resulted in an AUC of 0.68. The kinetic parameter SER reached a comparable AUC of 0.84. When combining these 6 parameters into a unified diagnostic model, the AUC was further improved to 0.86 . This combined classifier achieved a sensitivity of 0.81 and a specificity of 0.91 with a diagnostic accuracy of 0.87 (Table III).
Diagnostic performance of ADC value. A malignant lesion and a benign lesion can be seen in Fig. 2 and in Fig. 3, respectively. The box plot of the ADC for all patients is shown in Fig. 4. An overlap of ADC was observed between malignant and benign breast lesions. The mean ADC of malignant lesions (mean, $1.16 \times 10^{-3} \mathrm{~mm}^{2} / \mathrm{sec}$; $95 \% \mathrm{CI}, 1.13 \times 10^{-3} \mathrm{~mm}^{2} / \mathrm{sec}$; $1.20 \times 10^{-3} \mathrm{~mm}^{2} / \mathrm{sec}$ ) was significantly lower than that observed for benign lesions $\left(\mathrm{P}<0.05\right.$; mean, $1.68 \times 10^{-3} \mathrm{~mm}^{2} / \mathrm{sec} ; 95 \%$ CI, $\left.1.62 \times 10^{-3} \mathrm{~mm}^{2} / \mathrm{sec} ; 1.75 \times 10^{-3} \mathrm{~mm}^{2} / \mathrm{sec}\right)$. The predictive accuracy (sensitivity, specificity) of mean ADC was 0.84 (0.78 and 0.90 , respectively; Table III). Thus, a threshold value of $1.37 \times 10^{-3} \mathrm{~mm}^{2} / \mathrm{sec}$ can achieve a specificity of 0.90 , with a sensitivity of 0.78 , to discriminate between malignant and benign lesions.

Analysis of the model combining morphology and kinetic features with ADC values. When the ADC value was incorporated into the feature sets, the sensitivity, specificity and accuracy of the classification model increased to $0.85,0.94$ and 0.90 , respectively (Table III). The value of AUC was dramatically increased from 0.86 to 0.90 by incorporating without/with using ADC (Fig. 5). It implies that the ADC possesses the potential power in discriminating benign masses from malignant ones. It also suggests that addition of ADC 


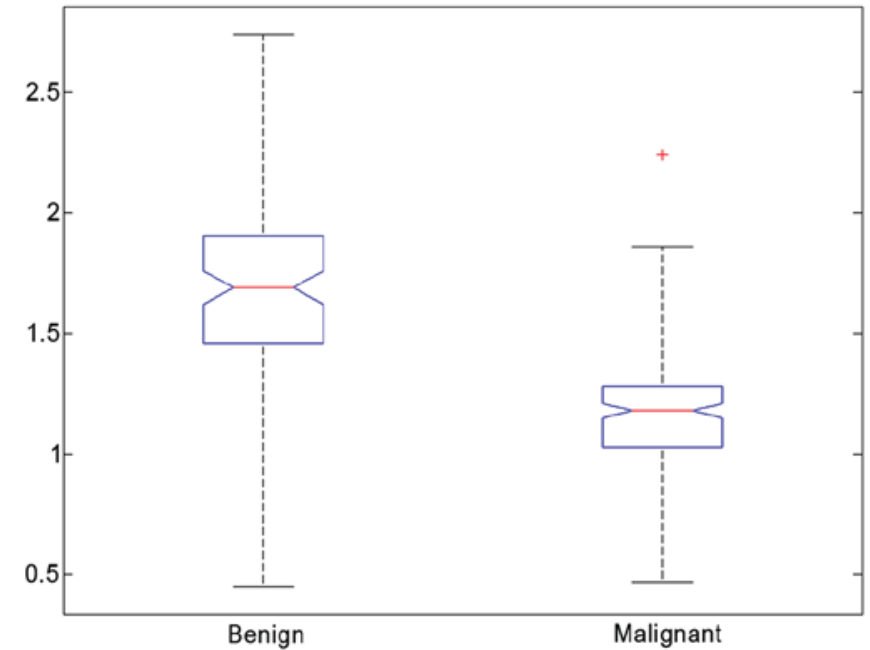

Figure 4. Box plot of ADC values of benign and malignant breast lesions, where the data presented as the median \pm interquartile range. Malignant breast lesions (mean ADC, $1.16 \times 10^{-3} \mathrm{~mm}^{2} / \mathrm{sec}$ ) exhibit lower mean ADCs than benign lesions (mean, $1.68 \times 10^{-3} \mathrm{~mm}^{2} / \mathrm{sec}, \mathrm{P}<0.05$ ). ADC, apparent diffusion coefficient.

values enhances the discriminatory power of the combined feature set.

\section{Discussion}

The results of the present study demonstrate that incorporating morphology and texture features, kinetic features and ADC into a multivariable diagnostic model in breast MRI increases diagnostic accuracy. Although previous studies have demonstrated that the combination of DWI and DCE-MRI can result in high diagnostic accuracy (28), and the addition of DWI to DCE-MRI improves the positive predictive value (PPV) of breast MRI (23), the assessments were mainly based on the subjective expertise of individual radiologists. In the current study, the undertaken contrast-enhanced breast images were analyzed by professional CAD software, which ensured less human interference and produced definitive parameters. A series of quantitative analysis was then followed to study the discrimination potentials of the images. El Khouli et al (29) demonstrated that quantitative assessment of the type of contrast enhancement kinetic curve on breast DCE-MRI for establishing or excluding malignancy is superior to standard qualitative assessment.

The present study applied quantitative analysis to characterize the morphology, texture and kinetic features of breast lesions and used advanced machine learning techniques to obtain a classifier for differential diagnosis. A total of 11 morphology, 13 GLCM texture and 2 kinetic features were extracted in order to characterize each lesion. Furthermore, 2 shape (heterogeneity, area) and 3 texture (sum variance, sum entropy, difference entropy) parameters and 1 kinetic parameter (SER) were selected by DWLH using ten-fold cross validation. The diagnostic performance based on 2 shape features (heterogeneity, area) and 3 texture features (sum variance, sum entropy, difference entropy) reached an AUC value of 0.68. Using the kinetic parameter SER it reached a comparable AUC of 0.84. When all 6 parameters were combined, the resulting AUC improved further to

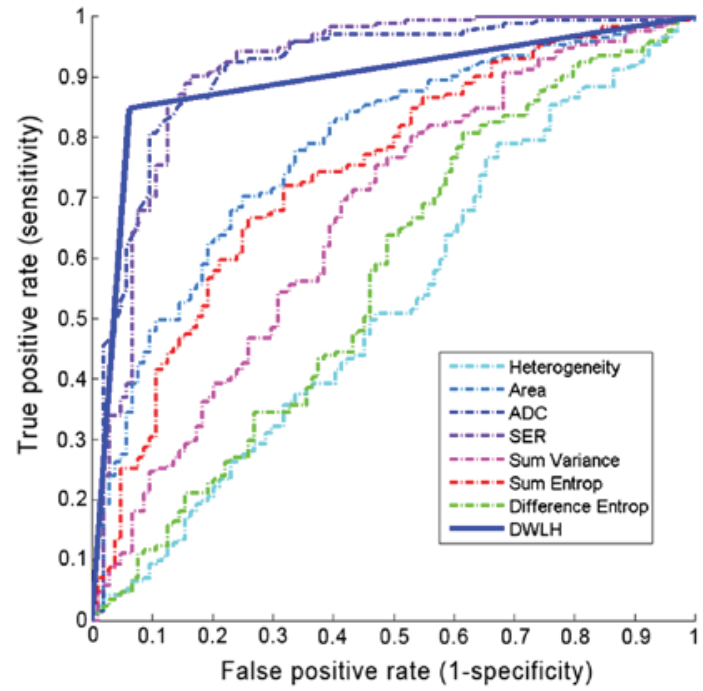

Figure 5. Comparison of ROC curves from morphological and kinetic analysis, from ADC values, and from combined features. The graph reveals that a model incorporating ADC values with morphological and kinetic features provides the best discriminative ability (AUC $=0.90)$, compared to individual features ( $\mathrm{AUC}=0.68$ for morphological features, $\mathrm{AUC}=0.84$ for kinetic features, and $\mathrm{AUC}=0.83$ for $\mathrm{ADC}$ values). $\mathrm{ROC}$, receiver operating characteristic; ADC, apparent diffusion coefficient; AUC, area under curve.

0.86. This finding demonstrates that the combination of the kinetic enhancement data and morphology information in a systematic model is the most effective and comprehensive approach to the diagnosis of breast masses. These results are consistent with those of Newell et al (28). The performance of the shape and textural features $(\mathrm{AUC}=0.68)$ in the present study is evidently lower than that of kinetic parameter SER (AUC=0.84), which is different from the results of Newell et al (28) who received an AUC of 0.87 when using morphological features to separate between benign and malignant breast masses and a comparable value of 0.88 when using the kinetic parameters.

In particular, it was found that SER was an effective kinetic feature for the diagnosis of breast masses. Its AUC value was the highest among all the parameters when measured individually. SER has the advantage of being able to depict the heterogeneous microvascular network in breast cancers (30). High SER corresponded to early signal enhancement, with rapid washout of intravenous contrast reflecting high tumor vascularity. Similarly, low SER correlated with slow and sustained enhancement of contrast, reflecting low tumor vascularity (31). SER, based on changes in signal intensity between 3 time points, is an effective kinetic parameter in the present study. However, it may be affected by the selection of time points, by the magnet strength, the sequence parameters (repetition time, echo time, flip angle) and by the contrast agent concentration. Therefore, with a different selection of sequence parameters, or a different selection of time points, different SER values could be derived, and the diagnostic effectiveness of SER may be altered.

Contrast-enhanced MRI imaging reflects the tumor vascular bed and ADC has been demonstrated to correlate with tumor cellularity density in breast cancer (32). The present results reveal that diagnostic accuracy is increased when the morphological and kinetic features are combined 
with the ADC value. This finding suggests that DWI may yield information different from and complementary to that obtained with DCE-MRI (33).

Additionally, the mean ADC of malignant lesions (mean, $\left.1.16 \times 10^{-3} \mathrm{~mm}^{2} / \mathrm{sec} ; 95 \% \mathrm{CI}, 1.13 \times 10^{-3} \mathrm{~mm}^{2} / \mathrm{sec}, 1.20 \times 10^{-3} \mathrm{~mm}^{2} / \mathrm{sec}\right)$ in the present study was significantly lower than that observed for benign lesions ( $\mathrm{P}<0.05$; mean, $1.68 \times 10^{-3} \mathrm{~mm}^{2} / \mathrm{se}$; $95 \% \mathrm{CI}$, $\left.1.62 \times 10^{-3} \mathrm{~mm}^{2} / \mathrm{sec} ; 1.75 \times 10^{-3} \mathrm{~mm}^{2} / \mathrm{sec}\right)$, which are similar results to other studies $(11,23)$. The diagnostic accuracy of DW imaging performed in the present study was 0.87 , which is slightly higher than previous studies $(33,34)$. It may be that the current study only evaluates breast mass lesions, while the prior studies evaluate MRI-detected mass and non-mass lesions. Besides, the present DW imaging protocol is different.

There were several limitations to the current study. The lesion segmentation was conducted in two-dimensions and high dimensional description is needed to provide rich spatial information. However, the segmentation algorithm was based on enhanced lesion images, and sequences of two-dimensional images can reflect spatial variations accurately. The present study also had technical limitations. The DW examinations were acquired with relatively thick slices $(5 \mathrm{~mm})$ in order to achieve an adequate signal-to-noise ratio. Partial volume averaging within imaging slices may affect the visibility of a number of lesions on DWI. This limitation could be overcome by utilizing longer scanning times or by imaging at higher field strengths (35). Finally, the current study excluded the non-mass lesions. This is as non-mass-like enhancement lesions exhibit poorly defined boundaries, which results in difficulty in the analysis of morphology. Diagnosis of non-mass lesions is much more challenging. However, kinetics and ADC analysis may be applied to characterize non-mass lesions. The evaluation of non-mass lesions may be an important next step.

To conclude, it was found that combining ADC with quantitative morphology, texture features and kinetic features improves the accuracy of characterization of breast lesions that present as a mass. These integrated characteristic parameters are promising in terms of generating an overall diagnostic marker for breast masses, which can achieve high levels of diagnostic accuracy.

\section{Acknowledgments}

Not applicable.

\section{Funding}

The present study was funded by the Science and Technology Planning Project of Guangdong Province, China (grant nos.2016B090918066,2016A010101013 and2017B020226004), the Science and Technology Program of Guangzhou, China (grant nos. 201704020060 and 201807010057), and the Health and Medical Collaborative Innovation Project of Guangzhou City (grant nos. 201604020003 and 201803010021).

\section{Availability of data and materials}

The datasets used and/or analyzed during the current study are available from the corresponding author on reasonable request.

\section{Authors' contributions}

XJ, FX and LZL performed data collection, data analysis, manuscript drafting and revision. YP aided in the image data collection and data analysis. HC and LL initially developed the concept of the study and contributed in writing the manuscript and all revisions. All authors read and approved the final manuscript.

\section{Ethics approval and consent to participate}

The study was approved by the Ethics Committee of Sun Yat-sen University Cancer Center (Guangzhou, China).

\section{Consent for publication}

All participants provided informed consent for the publication of this data.

\section{Competing interests}

The authors declare that they have no competing interests.

\section{References}

1. Sardanelli F, Boetes C, Borisch B, Decker T, Federico M, Gilbert FJ, Helbich T, Heywang-Köbrunner SH, Kaiser WA, Kerin MJ, et al: Magnetic resonance imaging of the breast: Recommendations from the EUSOMA working group. Eur J Cancer 46: 1296-1316, 2010.

2. Kuhl CK, Schrading S, Leutner CC, Morakkabati-Spitz N, Wardelmann E, Fimmers R, Kuhn W and Schild HH: Mammography, breast ultrasound, and magnetic resonance imaging for surveillance of women at high familial risk for breast cancer. J Clin Oncol 23: 8469-8476, 2005.

3. Schelfout K, Van Goethem M, Kersschot E, Colpaert C, Schelfhout AM, Leyman P, Verslegers I, Biltjes I, Van Den Haute J, Gillardin JP, et al: Contrast-enhanced MR imaging of breast lesions and effect on treatment. Eur J Surg Oncol 30: 501-507, 2004.

4. Tillman GF, Orel SG, Schnall MD, Schultz DJ, Tan JE and Solin LJ: Effect of breast magnetic resonance imaging on the clinical management of women with early-stage breast carcinoma. J Clin Oncol 20: 3413-3423, 2002.

5. Semiglazov V: Recist for response (clinical and imaging) in neoadjuvant clinical trials in operable breast cancer. J Natl Cancer Inst Monogr 2015: 21-23, 2015.

6. Iwasa H, Kubota K, Hamada N, Nogami M and Nishioka A: Early prediction of response to neoadjuvant chemotherapy in patients with breast cancer using diffusion-weighted imaging and gray-scale ultrasonography. Oncol Rep 31: 1555-1560, 2014 .

7. Jansen SA, Fan X, Karczmar GS, Abe H, Schmidt RA, Giger M and Newstead GM: DCEMRI of breast lesions: Is kinetic analysis equally effective for both mass and nonmass-like enhancement? Med Phys 35: 3102-3109, 2008.

8. Malich A, Fischer DR, Wurdinger S, Boettcher J, Marx C, Facius $M$ and Kaiser WA: Potential MRI interpretation model: Differentiation of benign from malignant breast masses. AJR Am J Roentgenol 185: 964-970, 2005.

9. Kuhl CK: MRI of breast tumors. Eur Radiol 10: 46-58, 2000.

10. Jiang Y, Nishikawa RM, Schmidt RA, Metz CE, Giger ML and Doi K: Improving breast cancer diagnosis with computer-aided diagnosis. Acad Radiol 6: 22-33, 1999.

11. Hatakenaka M, Soeda H, Yabuuchi H, Matsuo Y, Kamitani T, Oda Y, Tsuneyoshi M and Honda H: Apparent diffusion coefficients of breast tumors: Clinical application. Magn Reson Med Sci 7: 23-29, 2008.

12. Rubesova E, Grell AS, De Maertelaer V, Metens T, Chao SL and Lemort M: Quantitative diffusion imaging in breast cancer: A clinical prospective study. J Magn Reson Imaging 24: 319-324, 2006. 
13. Partridge SC, Rahbar H, Murthy R, Chai X, Kurland BF, DeMartini WB and Lehman CD: Improved diagnostic accuracy of breast MRI through combined apparent diffusion coefficients and dynamic contrast-enhanced kinetics. Magn Reson Med 65: 1759-1767, 2011.

14. Yabuuchi H, Matsuo Y, Okafuji T, Kamitani T, Soeda H, Setoguchi T, Sakai S, Hatakenaka M, Kubo M, Sadanaga N, et al: Enhanced mass on contrast-enhanced breast MR imaging: Lesion characterization using combination of dynamic contrast-enhanced and diffusion-weighted MR images. J Magn Reson Imaging 28: 1157-1165, 2008.

15. Shin K, Phalak K, Hamame A and Whitman GJ: Interpretation of breast MRI utilizing the bi-rads fifth edition lexicon: How are we doing and where are we headed? Curr Probl Diagn Radiol 46: 26-34, 2017.

16. Hylton NM: Vascularity assessment of breast lesions with gadolinium-enhanced MR imaging. Magn Reson Imaging Clin N Am 9: 321-332, 2001.

17. Kuhl CK, Mielcareck P, Klaschik S, Leutner C, Wardelmann E, Gieseke J and Schild HH: Dynamic breast MR imaging: Are signal intensity time course data useful for differential diagnosis of enhancing lesions? Radiology 211: 101-110, 1999.

18. Wu K: Analysis of parameter selections for fuzzy c-means. Pattern Recognit 45: 407-415, 2012.

19. Xu C and Prince JL: Snakes, shapes, and gradient vector flow IEEE Trans Image Process 7: 359-369, 1998.

20. Basu S, Goldgof D, Gu Y, Kumar V, Choi J, Gillies RJ, Hall OL and Gatenby RA: Developing a classifier model for lung tumors in CT-scan images. In: Proceedings of the IEEE International Conference on Systems, Man and Cybernetics, Anchorage, AK, pp1306-1312, 2011.

21. Fu LZB: A co-occurrence matrix algorithm used for medical image. In: Proceedings of the IEEE International Conference on Systems, Man and Cybernetics, Anchorage, AK, pp1318-1321, 2011.

22. Pang Y, Li L, Hu W, Peng Y, Liu L and Shao Y: Computerized segmentation and characterization of breast lesions in dynamic contrast-enhanced MR images using fuzzy c-means clustering and snake algorithm. Comput Math Methods Med 2012: 634907, 2012.

23. Partridge SC, DeMartini WB, Kurland BF, Eby PR, White SW and Lehman CD: Quantitative diffusion-weighted imaging as an adjunct to conventional breast MRI for improved positive predictive value. AJR Am J Roentgenol 193: 1716-1722, 2009.

24. Guyon IEA: An introduction to variable and feature selection. J Mach Learn Res 3: 1157-1182, 2003.

25. Cai H: Improvements over Adaptive Local Hyperplane to Achieve Better Classification. In: Advances in Data Mining. Applications and Theoretical Aspects. Perner P (ed). ICDM 2011. Lecture Notes in Computer Science, vol 6870. Springer, Berlin, Heidelberg, 2011.
26. Herrero JR and Navarro JJ: Exploiting computer resources for fast nearest neighbor classification. Pattern Anal Appl 10: 265-275, 2007.

27. Duan KB, Rajapakse JC, Wang H and Azuaje F: Multiple SVM-RFE for gene selection in cancer classification with expression data. IEEE Trans Nanobioscience 4: 228-234, 2005.

28. Newell D, Nie K, Chen JH, Hsu CC, Yu HJ, Nalcioglu O and Su MY: Selection of diagnostic features on breast MRI to differentiate between malignant and benign lesions using computer-aided diagnosis: Differences in lesions presenting as mass and non-mass-like enhancement. Eur Radiol 20: 771-781, 2010.

29. El Khouli RH, Macura KJ, Jacobs MA, Khalil TH, Kamel IR, Dwyer A and Bluemke DA: Dynamic contrast-enhanced MRI of the breast: Quantitative method for kinetic curve type assessment. AJR Am J Roentgenol 193: W295-W300, 2009.

30. Li KL, Partridge SC, Joe BN, Gibbs JE, Lu Y, Esserman LJ and Hylton NM: Invasive breast cancer: Predicting disease recurrence by using high-spatial-resolution signal enhancement ratio imaging. Radiology 248: 79-87, 2008

31. Esserman L, Hylton N, George $\mathrm{T}$ and Weidner $\mathrm{N}$ : Contrast-enhanced magnetic resonance imaging to assess tumor histopathology and angiogenesis in breast carcinoma. Breast J 5: 13-21, 1999.

32. Guo Y, Cai YQ, Cai ZL, Gao YG, An NY, Ma L, Mahankali S and Gao JH: Differentiation of clinically benign and malignant breast lesions using diffusion-weighted imaging. J Magn Reson Imaging 16: 172-178, 2002.

33. Partridge SC, Mullins CD, Kurland BF, Allain MD, DeMartini WB, Eby PR and Lehman CD: Apparent diffusion coefficient values for discriminating benign and malignant breast MRI lesions: Effects of lesion type and size. AJR Am J Roentgenol 194: 1664-1673, 2010.

34. Partridge SC, Demartini WB, Kurland BF, Eby PR, White SW and Lehman CD: Differential diagnosis of mammographically and clinically occult breast lesions on diffusion-weighted MRI. J Magn Reson Imaging 31: 562-570, 2010.

35. Rahbar H, Partridge SC, Demartini WB, Gutierrez RL, Allison KH, Peacock S and Lehman CD: In vivo assessment of ductal carcinoma in situ grade: A model incorporating dynamic contrast-enhanced and diffusion-weighted breast MR imaging parameters. Radiology 263: 374-382, 2012.

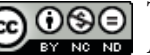

This work is licensed under a Creative Commons Attribution-NonCommercial-NoDerivatives 4.0 International (CC BY-NC-ND 4.0) License. 